\title{
Dynamic Evolution of New Grains in Magnesium Alloy AZ31 during Hot Deformation*
}

\author{
Xuyue Yang, Hiromi Miura and Taku Sakai \\ Department of Mechanical Engineering and Intelligent Systems, The University of Electro-Communications, Tokyo 182-8585, Japan
}

Dynamic grain evolution of a magnesium alloy AZ31 was studied in compression at $673 \mathrm{~K}\left(0.73 T_{\mathrm{m}}\right)$ by optical and SEM/OIM microscopy. The flow curve shows rapid hardening accompanied by a stress peak at a relatively low strain $\left(\varepsilon_{\mathrm{p}}=0.12\right)$, followed by strain softening and then a steady state flow stress at high strains. Fine grains evolved at corrugated grain boundaries at around $\varepsilon_{\mathrm{p}}$ and developed rapidly during strain softening, finally leading to a full structure of equiaxed fine grains. Such characteristics of new grain evolution and flow behavior are apparently similar to those in conventional, i.e. discontinuous, dynamic recrystallization (DRX). On the other hand, kink bands were observed frequently near corrugated grain boundaries and also in grain interiors, even around $\varepsilon_{\mathrm{p}}$. The misorientation of the boundaries of the kink bands increases rapidly during strain softening and approaches a saturation value of around $43^{\circ}$ at high strains. The average size of the regions fragmented by kink bands is almost the same as that of the new grains. It is concluded, therefore, that new grain evolution in this alloy is controlled by a deformation-induced continuous reaction, i.e. continuous DRX.

(Received November 7, 2002; Accepted December 9, 2002)

Keywords: magnesium alloy, hot deformation, new grain evolution, kink band, continuous dynamic recrystallization

\section{Introduction}

Magnesium (Mg) and its alloys show generally low ductility because of the few slip systems in the hexagonal close-packed (HCP) lattice and are categorized as hard plastic materials. Mg light-weight structural materials have been fabricated less frequently by plastic working such as rolling and other forming process than by a casting route. ${ }^{1,2)}$ It is expected, however, that under warm and hot deformation, several slip systems can be operated in addition to the basal slip system, leading to an increase of the plastic workability. It is also known in $\mathrm{Mg}$ alloys ${ }^{1)}$ that fine grains are developed at relatively low strains during warm and hot working. The plastic workability of fine-grained $\mathrm{Mg}$ alloys can be much improved by superplasticity. ${ }^{1}$ )

Fine grain evolution due to dynamic recrystallization (DRX) has been extensively studied in cubic metals with low to medium stacking fault energy (SFE), ${ }^{3,4)}$ However, there have been only a few studies on DRX in HCP metals. ${ }^{5-7)}$ It has been discussed in previous works ${ }^{5-7)}$ that dynamic evolution of new grains in $\mathrm{Mg}$ alloys can result from continuous DRX including twinning DRX and rotation DRX under cold and warm deformation. In contrast, discontinuous DRX including the nucleation of new grains and their growth takes place during deformation at high temperatures. There has been no clear explanation why the mechanisms of DRX change with deformation condition. It is also well known ${ }^{3,4)}$ that the dynamically recrystallized grain size in cubic metals is controlled only by deformation conditions, i.e. temperature and strain rate, irrespective of the initial grain size. It is found, however, that the dynamically recrystallized grain size in $\mathrm{Mg}$ alloys is clearly dependent not only on deformation conditions, but also on the initial grain size.,9)

In the present study, DRX behavior of a $\mathrm{Mg}-\mathrm{Al}-\mathrm{Zn}$ alloy was examined to clarify the microstructural changes during deformation at a temperature of $673 \mathrm{~K}$ (about $0.73 T_{\mathrm{m}}$, where

*This Paper was Originally Published in J. Japan Inst. of Light Metals 52 (2002).
$T_{\mathrm{m}}$ is the melting point). The nucleation site and growth process of DRX grains and texture development were studied in the $\mathrm{Mg}$ alloy. The mechanisms of DRX in the $\mathrm{Mg}$ alloy are discussed in detail.

\section{Experimental Procedure}

A commercially produced $\mathrm{Mg}$ alloy AZ31 was provided as a hot-extruded rod with a diameter of $19 \mathrm{~mm}$. The chemical composition was as follows; $\mathrm{Al} 2.68, \mathrm{Zn} \mathrm{0.75,} \mathrm{Mn} \mathrm{0.68,} \mathrm{Cu}$ 0.001 , Si 0.003, Fe 0.003, and balance $\mathrm{Mg}$ (all in mass\%). Cylindrical samples of $8 \mathrm{~mm}$ in diameter and $12 \mathrm{~mm}$ in height were machined from the rod parallel to the extrusion direction. Then 6 evenly spaced annular grooves of $0.12 \mathrm{~mm}$ depth were machined into the end faces of cylinders in order to promote the retention of a graphite lubricant (DAG 154). The samples were annealed at $733 \mathrm{~K}$ for $7.2 \mathrm{ks}$ and then furnace cooled, leading to the evolution of equiaxed grains with an average size of about $22 \mu \mathrm{m}$ (see Fig. 1(a)).

Compression tests were carried out at constant true strain rates on a testing machine equipped with a water quenching apparatus, which made it possible to quench the samples within $1.5 \mathrm{~s}$ after hot deformation was ceased. The samples were inserted into the preheated vessel at a test temperature of $673 \mathrm{~K}$ and kept for $0.9 \mathrm{ks}$ in vacuum of about $10^{-1} \mathrm{~Pa}$, followed by compression at a true strain rate of $3 \times 10^{-3} \mathrm{~s}^{-1}$. Each deformed sample was cut along a plane parallel to the compression axis, mechanically polished, electropolished in a solution of nitric acid, glycerol and ethanol with a $1: 3: 6$ volume ratio, and then chemically etched in a $6 \%$ picric acid in methanol. The metallographic analysis was carried out by optical microscopy (OM) and scanning electron microscopy (SEM) with orientation imaging microscopy (OIM).

A selection of samples were mechanically polished before deformation and scratched parallel to the compression axis by means of diamond paste to form marker lines of about $0.05 \mu \mathrm{m}$ in width. The surface morphologies developed were examined by $\mathrm{OM}$ and SEM/OIM. 


\section{Results and Discussion}

\subsection{Microstructural changes with deformation}

A typical true stress-true strain $(\sigma-\varepsilon)$ curve and microstructural changes with straining at $673 \mathrm{~K}$ and at $3 \times 10^{-3} \mathrm{~s}^{-1}$ are represented in Fig. 1. Rather smooth grain boundaries of the initial structure (Fig. 1(a)) are frequently corrugated and fine grains are evolved near the boundaries at $\varepsilon=0.10$ (Fig. 1(b)). The peak strain, where the stress peak appears, was $\varepsilon_{\mathrm{p}}=0.12$. At a strain of 0.3 , where strain softening takes place, new fine grains are developed in a colony along most of the grain boundaries (Fig. 1(c)). Further straining to around 0.5 , where a steady state flow starts to take place, equiaxed new grains are developed homogeneously and almost fill the whole microstructure (Fig. 1(d)). The results in Fig. 1 are almost the same as the previous ones reported by Ion et al. $^{5)}$ and Galiev et al. ${ }^{7)}$ The close relationship between the flow behavior and the development of a new grain structure is similar to that of conventional DRX in cubic metals. ${ }^{3,4)}$

Surface morphologies of the sample deformed to strains of 0.1 and 0.25 at $673 \mathrm{~K}$ and at $3 \times 10^{-3} \mathrm{~s}^{-1}$, are shown in Fig. 2. It can be seen that grain boundaries are corrugated and migrate locally by a limited distance at $\varepsilon=0.1$. It is remarkable to note in Fig. 2(a) that deformation bands are developed in some grain interiors. Such bands have been called kink bands in $\mathrm{Mg}$ alloys. ${ }^{10,11)}$ Their presence is
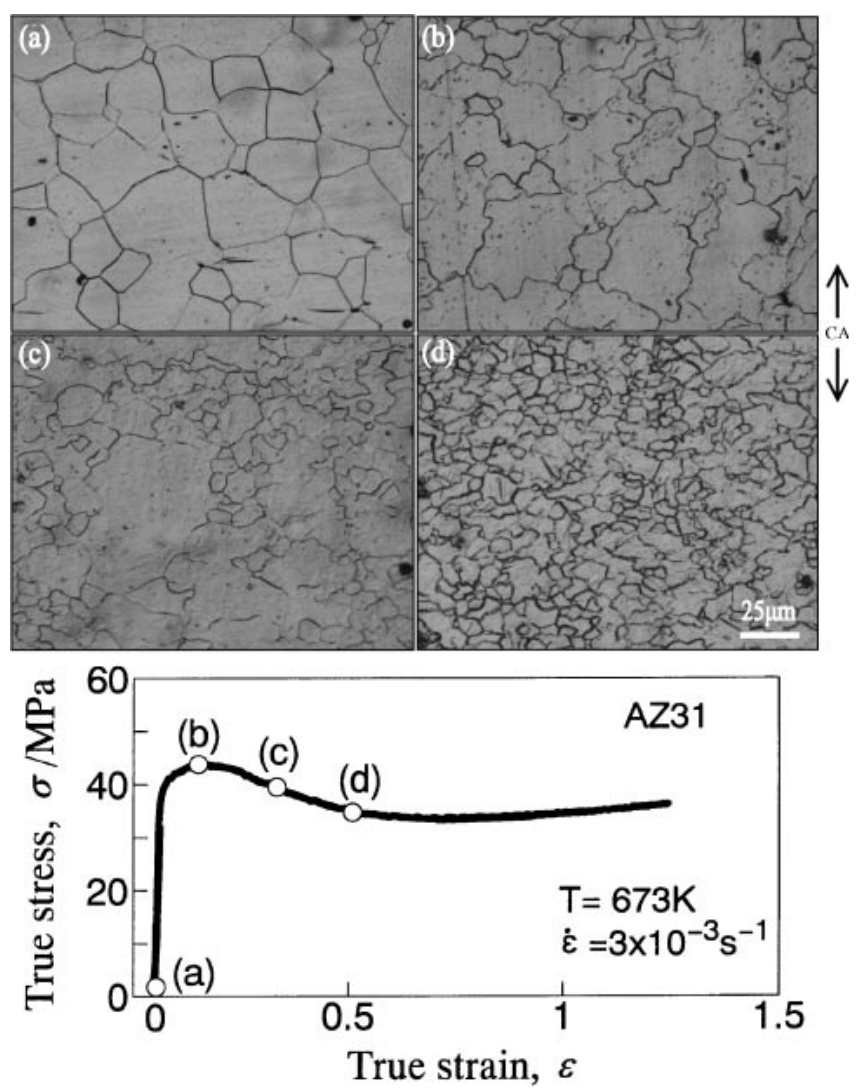

Fig. 1 Optical microstructures and typical true stress-true strain $(\sigma-\varepsilon)$ curve of AZ31 alloy deformed to various strains at $673 \mathrm{~K}$ and at $3 \times 10^{-3} \mathrm{~s}^{-1}$, followed by water quenching. The compression axis (CA) was parallel to the extrusion direction of the alloy. (a) $\varepsilon=0$, (b) $\varepsilon=0.1$, (c) $\varepsilon=0.3$ and (d) $\varepsilon=0.5$.

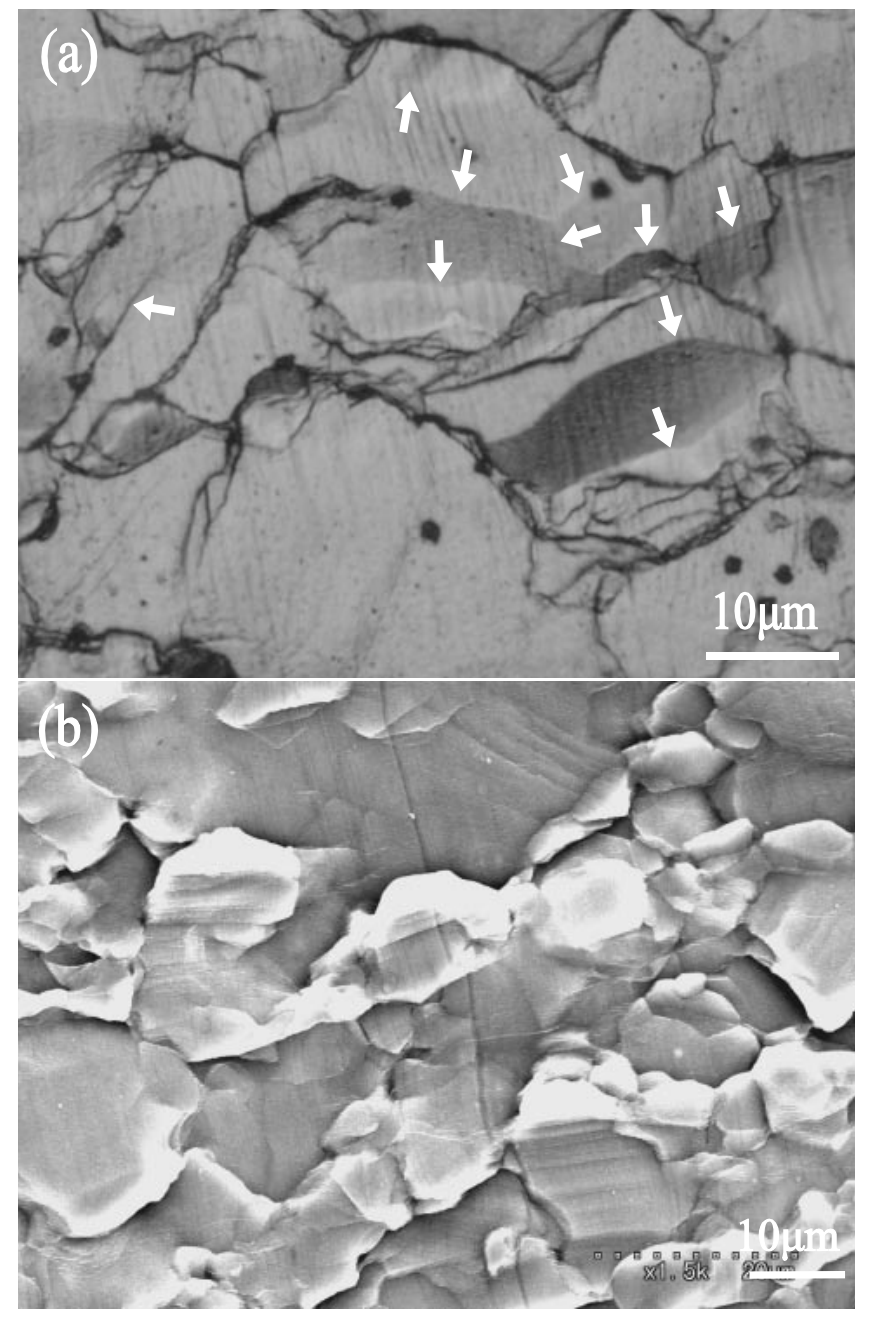

Fig. 2 Surface morphology of AZ31 alloy deformed to (a) $\varepsilon=0.1$ (OM) and (b) $\varepsilon=0.25(\mathrm{SEM})$ at $673 \mathrm{~K}$ and at $3 \times 10^{-3} \mathrm{~s}^{-1}$ (unetched). Note the development of local migration of corrugated grain boundaries and deformation bands (kink bands) in grain interiors in (a), and the occurrence of fine grain evolution, grain boundary sliding and grain rotation in (b).

confirmed in Figs. 3 and 4. At a strain of 0.25, fine grains are evolved frequently along the original grain boundaries. Grain boundary sliding and grain rotation also takes place. This is confirmed by the displacement of scratched marker lines as well as the grain boundary appearance itself. No microscopic voids nor cracks were observed even after straining to more than $\varepsilon=0.5$ (see Fig. 1).

\subsection{Dynamic nucleation of new grains}

It was clearly seen in Figs. 1 and 2 that corrugated grain boundaries migrate by a limited distance accompanied with grain boundary sliding and grain rotation. Kink bands are also developed in some grain interiors at $\varepsilon \approx 0.1$. Under such conditions new grains are evolved partly along the corrugated grain boundaries. A typical OIM micrograph is shown in Fig. 3. The sample was deformed to $\varepsilon=0.1$ under the same test condition as in Figs. 1 and 2. Different colors indicate different crystallographic orientations defined in the inverse pole figure. In Fig. 3, high-angle boundaries with misorientations of more than $15^{\circ}$ are delineated by thick-black lines, while low-angle boundaries in the range of $4^{\circ}$ and $15^{\circ}$ by thin-black lines and those in the range $2^{\circ}-4^{\circ}$ by white lines. 


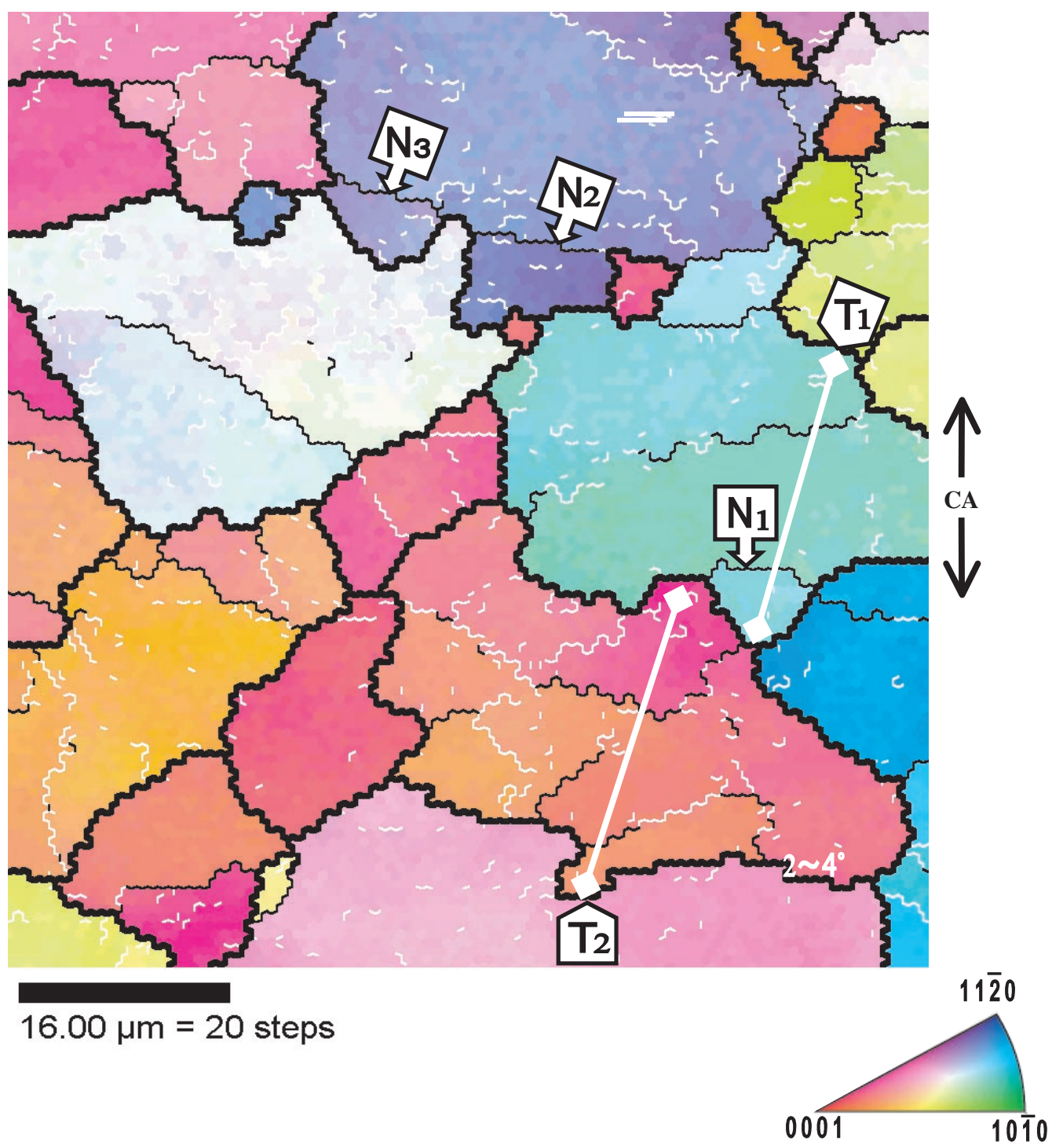

Fig. 3 Typical OIM map of AZ31 alloy deformed to a strain of 0.1 at $673 \mathrm{~K}$ and at $3 \times 10^{-3} \mathrm{~s}^{-1}$. Thin white lines correspond to boundaries of misorientation $>2^{\circ}$ thin black line $>4^{\circ}$ and thick black lines $>15^{\circ}$, respectively. Colors correspond to crystallographic orientations indicated in the inverse pole figure.

It can be seen clearly in Fig. 3 that a few fine grains are evolved along corrugated grain boundaries, and new boundaries with medium angle misorientations are developed in some regions near the corrugated boundaries, as indicated by $\mathrm{N}_{1}, \mathrm{~N}_{2}$ and $\mathrm{N}_{3}$. These places may be the nucleation sites of new grains, as discussed in the next section. Such a process of new grain formation may be similar to that in conventional DRX in cubic metals, i.e. the bulging out of part of the serrated grain boundaries. ${ }^{12-14)}$ It is also of note in Fig. 3 that new boundaries with low to medium angle misorientations are developed in several grain interiors and some of them intersect with each other. These new boundaries are considered to correspond to those in the kink bands shown in Fig. 2(a), and can be similar to transition bands or microbands. ${ }^{11)}$ It is concluded in Fig. 3 that several initial grains are fragmented by the formation of such kink bands and the crystal orientation of each fragmented region is slightly changed in a grain interior at $\varepsilon=0.1$.

The point-to-point misorientation $(\theta)$ and the cumulative misorientation $(\Sigma \theta)$ were measured along the lines $\mathrm{T}_{1}$ and $\mathrm{T}_{2}$ indicated in Fig. 3. The $\theta$ and the $\Sigma \theta$ values give the relative difference of crystal orientation between two adjacent scan points $(0.4 \mu \mathrm{m})$ and between a first scan point near a grain boundary and a point-n in another one in the opposite side, respectively. Figures 4(a) and (b) show the results for $\theta$ shown by the solid line and for $\Sigma \theta$ by a mark $(\square)$ measured along the lines $\mathrm{T}_{1}$ and $\mathrm{T}_{2}$. It is interesting to note that $\theta$ ranges from $2^{\circ}$ to $3^{\circ}$, but exceeds $6^{\circ}$ at several places. $\Sigma \theta$ changes discontinuously at the same places which correspond to the boundaries of kink band. Figure 4(c) shows the variation of $\theta$ and $\Sigma \theta$ developed in a typical grain deformed to $\varepsilon=0.15$. The value of $\theta$ for the boundaries of the kink band rises from about $6^{\circ}$ to above $12^{\circ}$ and new kink bands are evolved by deformation from $\varepsilon=0.1$ to 0.15 . On the other hand, gradients of lattice orientation (i.e. the variations of $\Sigma \theta$ ) are within a few degrees near both the new and the original grain boundaries. This is in contrast with conventional DRX in cubic metals, i.e. high orientation and high strain gradients are formed near the grain boundaries, leading to nucleation due to bulging followed by the growth of new grains. ${ }^{11-14)}$ 


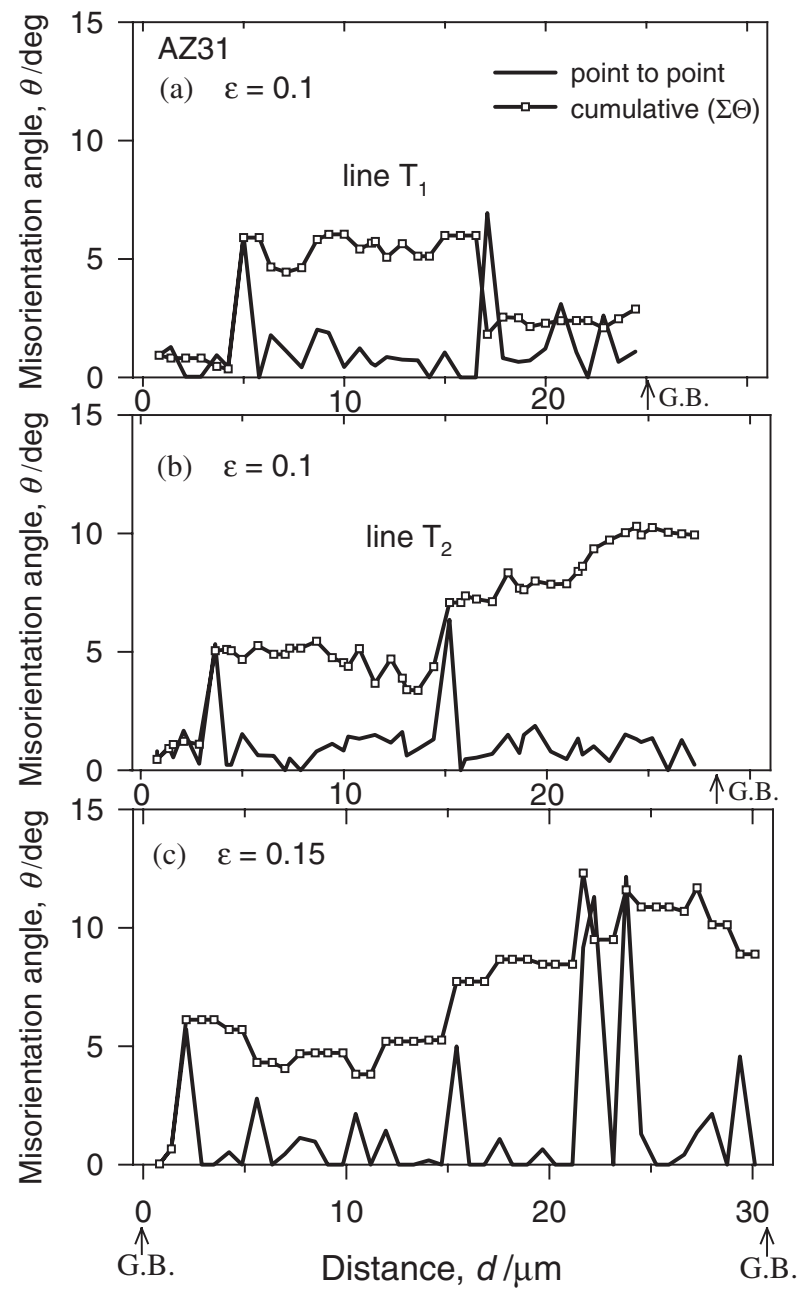

Fig. 4 Point-to-point misorientations $\theta$ (solid line) and cumulative misorientations $\Sigma \theta(\square)$ measured along the lines (a) $\mathrm{T}_{1}$ and (b) $\mathrm{T}_{2}$ marked in Fig. 3. The data for the sample deformed to 0.15 are also shown for comparison in (c). G.B. represents grain boundary.

\subsection{Progress of new grain formation}

Typical OIM micrographs are shown in Fig. 5 for the sample deformed to 0.3 and 0.5 at $673 \mathrm{~K}$ and at $3 \times 10^{-3} \mathrm{~s}^{-1}$. With increasing strain from $\varepsilon=0.1$ to 0.5 , where work softening takes place, the fraction of new grains increases rapidly and equiaxed new grains are developed homogeneously in $\varepsilon>0.5$ throughout the material. As can be seen in the colors in Fig. 5(b), crystal orientations of new grains are almost randomly distributed. On the other hand, it is remarkable in Figs. 5(a) and (b) that some rather coarser grains remain in the region of fine grains. New boundaries related to kink bands are scarcely developed in these coarser grains.

Figure 6 shows changes in the misorientation distribution of (sub)grain and transition boundaries related to kink band with increasing deformation. The distribution was measured in a unit area of $20 \times 20 \mu \mathrm{m}^{2}$ which is roughly within an initial grain interior. At a strain of 0.1 (Fig. 6(a)), some high angle boundaries detected may be the initial grain boundaries. Most of the boundaries related to kink bands and (sub)grains have misorientation of less than $10^{\circ}$ at $\varepsilon=0.1$. They change from low to medium and high angle boundaries during the progress of work softening (Figs. 6(b) and (c)), leading to a rapid increase in the average misorientation and a decrease in the fraction of low angle boundaries. With further straining to more than 0.5 , the average value of the misorientation approaches a constant value of about $43^{\circ}$.

Changes, with increasing deformation, of the average misorientation $\left(\theta_{\mathrm{av}}\right)$ obtained in Fig. 5 and the volume fraction of new grains $\left(X_{\mathrm{v}}\right)$ measured in Figs. 1 and 5 are depicted in Fig. 7. Both $\theta_{\mathrm{av}}$ and $X_{\mathrm{v}}$ start to increase at around the $\varepsilon_{\mathrm{p}}(=0.12)$ and rise rapidly during work softening, finally approaching saturation values at high strains, i.e. about $43^{\circ}$ and 0.85 , respectively. Such behaviors of $\theta_{\mathrm{av}}$ and $X_{\mathrm{v}}$ are considered to correspond to the shape of the flow curve in Fig. 1 as well as the development of an equiaxed new grain structure at $\varepsilon>0.5$ (Figs. 1(d) and 4(b)). It is interesting to note in Fig. 7(b) that new grains are not fully developed even under a steady state flow. Such a result has not been reported in conventional DRX of cubic metals. ${ }^{3,4)}$

Figure 8 shows the strain dependence of the average grain sizes measured over the whole map area $\left(D_{\text {av }}\right)$ including new and old grains as well as those measured in the regions of dynamically developed grains $\left(D_{\text {rex }}\right)$. The $D_{\text {av }}$ drops monotonously with strain and approaches a constant value of $10 \mu \mathrm{m}$ at high strains. On the other hand, the $D_{\text {rex }}$ increases a little from 7.8 to $9.3 \mu \mathrm{m}$ and so is considered to be roughly constant during deformation. Such results may be similar to those for necklace DRX in cubic metals. ${ }^{3)}$ By the way, the average size of the regions fragmented by kink bands at $\varepsilon=$ 0.1 was measured in Fig. 3 and is plotted in Fig. 8. It is interesting to note that the average size of fragmented regions at low strains is roughly the same as that of $D_{\text {rex }}$ at high strains. This suggests that each fragmented region surrounded by low to medium angle boundaries may transform to a new grain with high angle ones during deformation.

\subsection{Mechanism of DRX in Mg alloy}

It was proved by the present results described above that the high temperature flow behavior of $\mathrm{Mg}$ alloy AZ31 is closely related to dynamic formation of new grains due to the operation of DRX. Their characteristics are generally similar to those of conventional DRX behavior appearing in cubic metals. ${ }^{3,4)}$ Namely, the initial grain boundaries are frequently corrugated at around the peak strain and some new boundaries with medium angle misorientations are formed in regions near the corrugated boundaries. During work softening, new grains are developed in a colony formation along the grain boundaries and finally equiaxed new grains are developed throughout the material at high strains. Some of the results for the $\mathrm{Mg}$ alloy are, however, not the same as those of conventional DRX taking place in cubic metals. For example, kink bands are frequently formed in grain interior even at low strains. In other words, initial grains are fragmented by the boundaries of kink bands formed at early stages of deformation. Also, dynamically evolved grains are not fully developed during a steady state flow. (Figs. 4 and $6)$.

The process of DRX nucleation taking place in the present $\mathrm{Mg}$ alloy will be discussed here based on the current results and discussion. With early deformation, initial grain boundaries migrate locally and are frequently corrugated. Kink bands are concurrently formed in original grain interiors and 


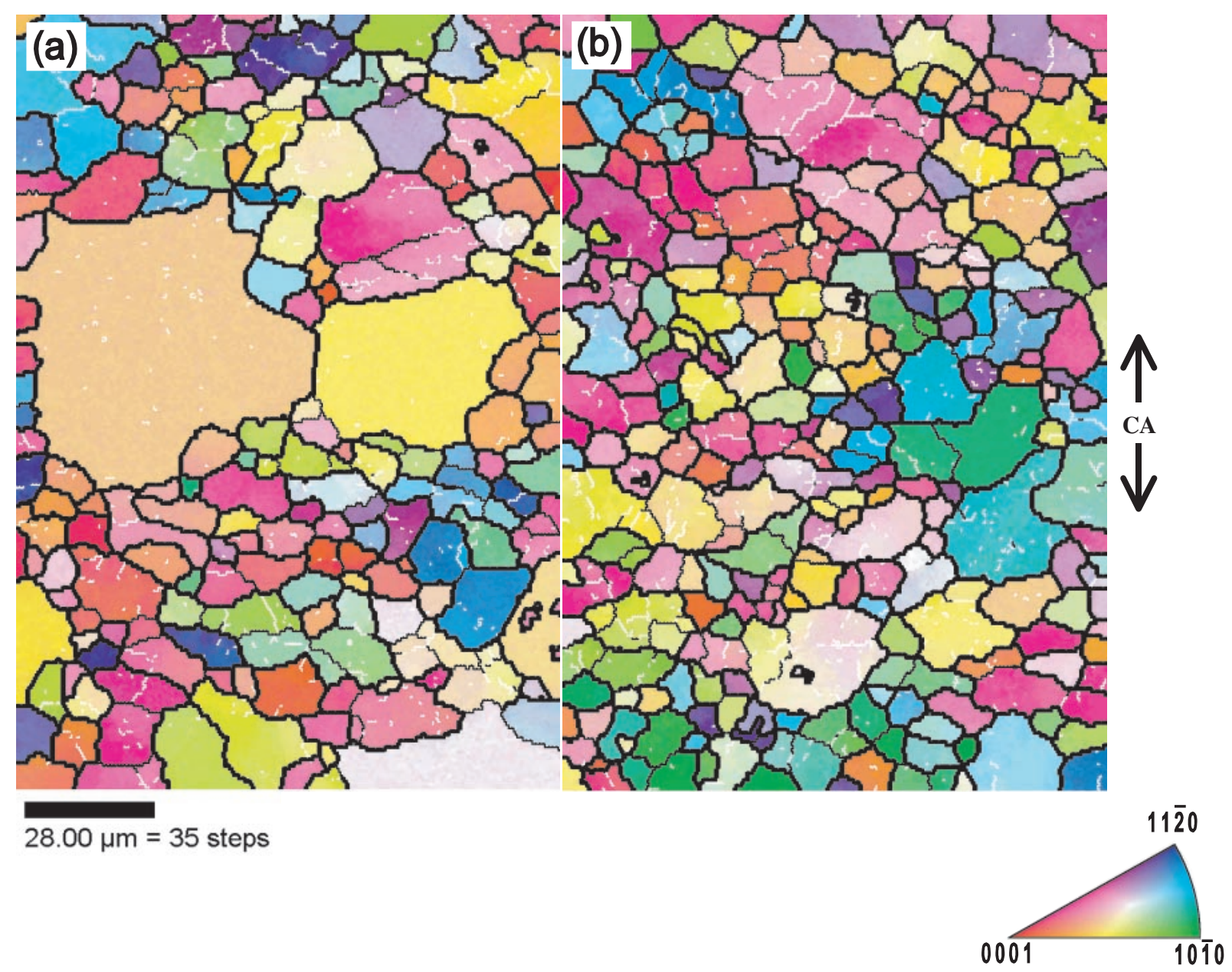

Fig. 5 Typical OIM maps of AZ31 alloy deformed to strains of (a) 0.3 and (b) 0.5 at $673 \mathrm{~K}$ and at $3 \times 10^{-3} \mathrm{~s}^{-1}$. The colors and lines indicate the same meanings as in Fig. 3.

in the regions of corrugated grain boundaries. They may form to satisfy grain compatibility requirements, perhaps because $\mathrm{Mg}$ has a limited number of active slip systems. The misorientation and the number of the boundaries of kink bands rise rapidly with further deformation. Finally, the regions fragmented by kink bands are bounded by high angle boundaries, resulting in evolution of new grains assisted by dynamic recovery and also grain boundary sliding (Fig. 2(b)).

These new grains can hardly grow in $\mathrm{Mg}$ alloy under hot deformation for the following reasons. First, only small orientation and strain gradients are developed near the boundaries of original grains and kink bands (Fig. 4). The average size of the fragmented regions is roughly the same as the DRX grain size (Fig. 8). This indicates that there is limited opportunity for long distance growth of each DRX grain. It is concluded, therefore, that the dynamic formation of new grains discussed above can result from a series of strain-induced continuous reactions, that are essentially similar to continuous (or in-situ) DRX.

Continuous DRX is essentially a one-step phenomenon, i.e. new grains are nucleated homogeneously throughout the material and can hardly grow. In contrast, conventional (discontinuous) DRX involves a two-step process, i.e. the nucleation of new grains surrounded by high angle boundaries followed by their long distance migration. ${ }^{15)}$ Some previous studies on the hot deformation of $\mathrm{Mg}$ alloys ${ }^{5-7}$ ) concluded that new grain development results from conventional (discontinuous) DRX. This arises because the formation of new grains and the flow behavior of $\mathrm{Mg}$ alloy are apparently similar to those for DRX behavior of general cubic metals, as described above.

It should be noted that in DRX of the present Mg alloy new grains were not fully developed throughout the material, even at high strains (Figs. 5 and 7). When original grains are surrounded by new fine grains, kink bands may be hardly developed in the remained original grains, because constrained deformation by surrounding grains can be relaxed by frequent operation of grain boundary sliding and dynamic recovery in the surrounding fine grains. New grains developed by continuous DRX have a limited potential for significant growth, because only small orientation and strain gradients are developed near the boundaries.

\subsection{Work softening due to texture change}

It was concluded in the previous section that new grain formation in the current $\mathrm{Mg}$ alloy can be explained by continuous DRX. The restoration mechanism operating in continuous DRX is principally dynamic recovery. ${ }^{11,16)}$ The $\sigma-\varepsilon$ curve controlled by dynamic recovery generally shows no work softening and only a steady state flow at high strains. This conflicts with the present $\sigma-\varepsilon$ curve, which shows work softening (Fig. 1). It is well known ${ }^{1,5)}$ that $\mathrm{Mg}$ extruded rod 


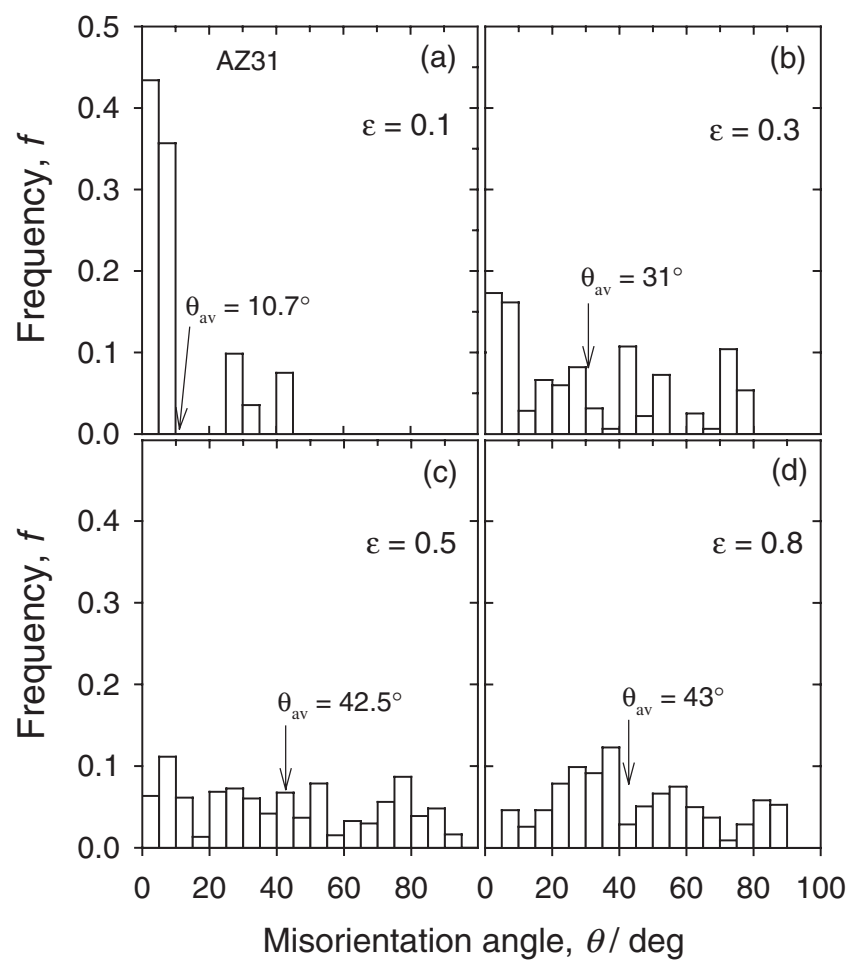

Fig. 6 Misorientation distributions of dislocation and (sub)grain boundaries developed in an unit area of $20 \times 20 \mu \mathrm{m}^{2}$, which is roughly within an initial grain interior.

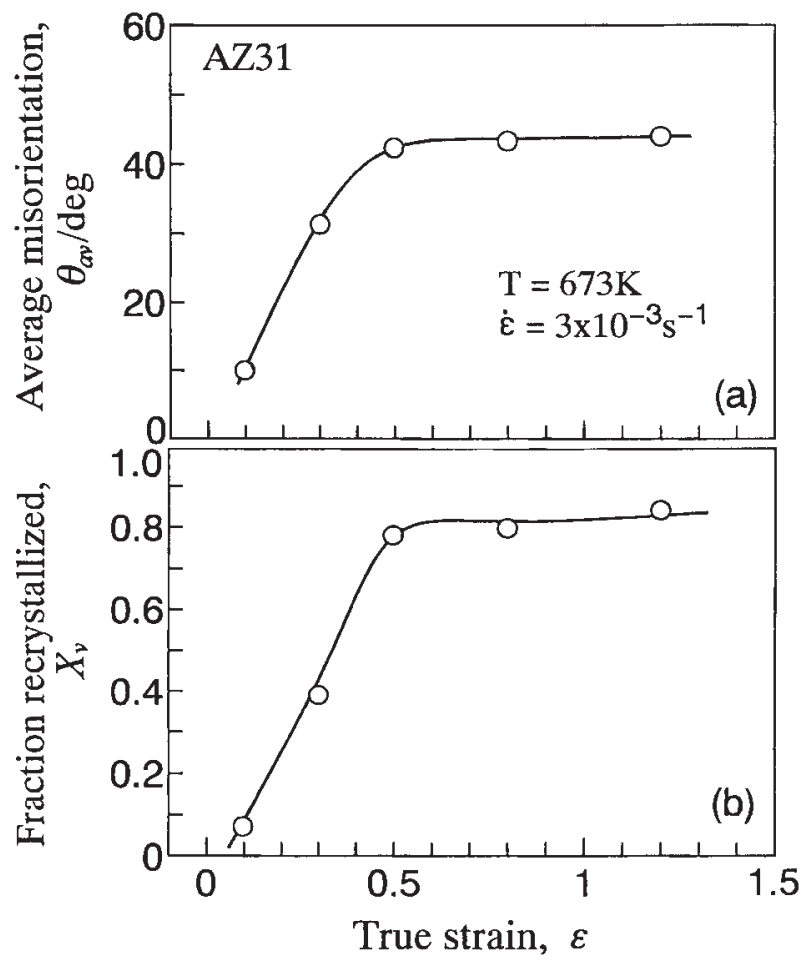

Fig. 7 Strain dependence of (a) average misorientation of dislocation and (sub)grain boundaries, $\theta_{\mathrm{av}}$, and (b) fraction dynamically recrystallized, $X_{\mathrm{v}}$, of AZ31 alloy deformed at $673 \mathrm{~K}$ and at $3 \times 10^{-3} \mathrm{~s}^{-1}$.

has a strong texture in which the basal plane of the HCP lattice lies parallel to the extrusion direction (and so the compression direction), as confirmed in Fig. 9.

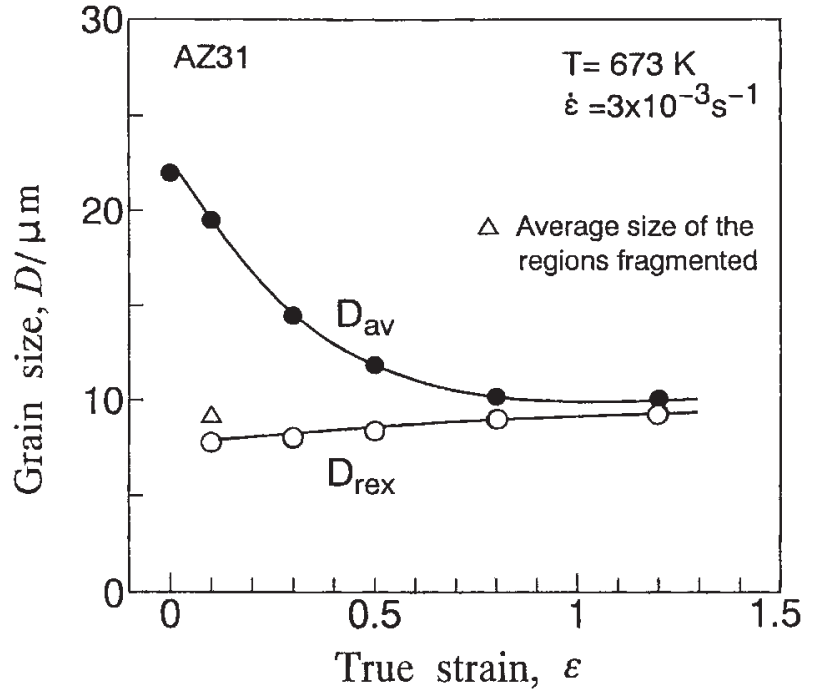

Fig. 8 Strain dependence of average grain sizes in the whole area, $D_{\mathrm{av}}$, and that in dynamically recrystallized regions, $D_{\text {rex }}$, of AZ31 alloy deformed at $673 \mathrm{~K}$ and at $3 \times 10^{-3} \mathrm{~s}^{-1}$. Average size of the regions fragmented by kink band at $\varepsilon=0.1$ is also shown for comparison.

\begin{tabular}{|c|c|c|}
\hline Strain & TD & ND \\
\hline 0 & $\left.\overbrace{0001}^{5.6}\right|_{1010} ^{1120}$ & $\sum_{0001}^{2.6}$ \\
\hline 0.3 & $\frac{2.9}{0001}$ & $\sum_{0001}^{1120}$ \\
\hline 0.5 & 0.87 & $\sum_{0001}^{1.5}$ \\
\hline 0.8 & $\sum_{0001}^{0.8}$ & $\underset{0001}{3.2^{2.6^{2}}}$ \\
\hline
\end{tabular}

Fig. 9 Strain dependence of inverse pole figures of the deformation texture developed in AZ31 alloy in tangential (TD) and normal direction (ND) of the cylindrical samples. The samples, prepared of the $\mathrm{Mg}$ rod parallel to the extrusion direction, were deformed in compression to various strains at $673 \mathrm{~K}$ and at $3 \times 10^{-3} \mathrm{~s}^{-1}$.

Changes in the inverse pole figure for tangential direction (TD) and normal (i.e. compression) direction (ND) of the cylindrical sample with compression at $673 \mathrm{~K}$ and at $3 \times 10^{-3} \mathrm{~s}^{-1}$ are shown in Fig. 9. The basal plane rotates 
gradually from around $0^{\circ}$ to near $90^{\circ}$ to the compression axis with deformation. It is possible, therefore, that work softening in the present $\mathrm{Mg}$ alloy does not result from new grain development, but rather from a change in the basal plane from the hard slip directions to the soft or stable ones, that is, geometric softening occurs. For confirmation of this idea, a cylindrical sample was machined from the extruded rod of AZ31 alloy with the axis aligned along the radial direction, and compressed at $673 \mathrm{~K}$ and at $3 \times 10^{-3} \mathrm{~s}^{-1}$. The details of the results are described in elsewhere ${ }^{17)}$ but the $\sigma-\varepsilon$ curve showed no appreciable work softening and only a steady state flow following a work hardening at relatively low strains. The evolution process of new grains and the average grain size developed in this sample were, however, almost the same as those in the present work mentioned above. The basal slip plane lying perpendicular to the compression axis was scarcely changed by compression. ${ }^{17)}$

\section{Conclusions}

Fine grain evolution taking place during hot deformation of a magnesium alloy AZ31 was studied in compression at $673 \mathrm{~K}\left(0.73 T_{\mathrm{m}}\right)$ by optical and SEM/OIM microscopy. The main results obtained are summarized as follows.

(1) The flow curve shows a rapid hardening and a stress peak at a relatively low strain $\left(\varepsilon_{\mathrm{p}}=0.12\right)$, followed by strain softening and then a steady state flow at high strains. Fine grains are evolved at corrugated grain boundaries at around $\varepsilon_{\mathrm{p}}$ and developed rapidly during strain softening, finally leading to an almost full evolution of equiaxial fine grains.

(2) Kink bands are evolved frequently at corrugated grain boundaries and also in grain interiors at around $\varepsilon_{\mathrm{p}}$. The misorientation of the boundaries of kink band increases rapidly during strain softening and approach a saturation value of around $43^{\circ}$ in high strain.

(3) The average size of new grains scarcely changes during deformation and is almost the same as that of the regions fragmented by kink band.

(4) It is concluded from the above results, (2) and (3), that new grain evolution can be controlled by a series of strain-induced continuous reactions, i.e. continuous dynamic recrystallization (DRX).

(5) Work softening taking place in the present $\mathrm{Mg}$ alloy does not result from new grain formation due to continuous DRX, but from a change in basal plane from the hard slip directions to the soft or stable ones, that is, geometric softening occurs.

\section{Acknowledgments}

The authors wish to acknowledge Prof. K.Higashida of Kyushu University, Japan, and Dr. M. R. Barnett of Deakin University, Australia, for stimulation discussion of formation of kink band and DRX in Mg alloy. They also intended to the following bodies for the financial support: Ministry of Education, Science and Culture and the Light Metals Educational Foundation, Japan.

\section{REFERENCES}

1) Y. Chino and M. Mabuchi: J. Jpn Inst. of Light Metals. 51 (2001) 498502.

2) H. Friedrich and S. Schuman: J. Mater. Process. Tech. 117 (2001) 276281.

3) T. Sakai and J. J. Jonas: Acta Metall. 32 (1984) 189-209.

4) T. Sakai: Thermomechanical Processing of Steels (J. J. Jonas Symposium), eds. S. Yue and E. Essadiqi, (TMS-CIM, Montreal, 2000) pp. 47-62.

5) S. E. Ion, F. J. Humphreys and S. H. White: Acta Metall. 30 (1982) 1909-1919.

6) R. Kaibyshev and O. Sitdikov: Recrystallization and Related Phenomena (ReX'96), ed by T. R. McNelley, (MIAS, Monterey, 1997) pp. 287-294.

7) A. Galiyev, R. Kaibyshev and G. Gottstein: Acta Mater. 49 (2001) 1199-1207.

8) R. O. Kaibyshev, A. M. Galiev and B. K. Sokolov: Phys. Met. Metall. 78 (1994) 209-217.

9) H. Watanabe, H. Tsutsui, T. Mukai, K. Ishikawa, Y. Okanda, M. Kohzu and K. Higashi: Mater. Trans. 42 (2001) 1200-1205.

10) K. Higashida, J. Takamura and N. Narita: Mater. Sci. Eng. 81 (1986) 239-258.

11) F. J. Humphreys and M. Hatherly: Recrystallization and Related Annealing Phenomena, (Pergamon, 1995) pp. 19-38, pp. 167-171.

12) H. Miura, H. Aoyama and T. Sakai: J. Japan Inst. Metals 58 (1994) 267-275.

13) A. Belyakov, H. Miura and T. Sakai: Mater. Sci. and Eng. A255 (1998) 139-147.

14) A. M. Wusatowska-Sarnek, H. Miura and T. Sakai: Mater. Sci. Eng. A323 (2002) 177-186.

15) T. Sakai and J. J. Jonas: Encyclopedia of Materials: Science and Technology, eds. K. H. J. Buschow et al., (Elsevier, Oxford, 2001) pp. 7079-7084.

16) A. Belyakov, T. Sakai, H. Miura and K. Tsuzaki: Philos. Mag. A81 (2001) 2629-2643.

17) X. Yang, H. Miura and T. Sakai: Light Metals 2002 Metaux Lergers (including H. J. McQueen Symposium), ed. T. Lewis (TMS-CIM, Montreal, 2002), pp. 867-878. 